\title{
And a good time was had by all!
}

A S I write this, the American Thoracic Society 2000 meeting held in Toronto has been over for a week. From every point of view it seems to have been a great success. While having the drawbacks of very large meetings, with some tiresome separation between different sessions, one could not complain about the depth of coverage, with over 6000 poster and slide presentations. I was particularly impressed with the number of senior researchers who took part and demonstrated amply that they were not merely names from the past but had much to offer research fellows, especially on a one-to-one basis. One could find standing in front of a poster - Sol Permutt, Bob Hyatt, John West, Ann Woolcock, Neil Pride and many others who gave freely of their time, experience and wisdom. There can be no better meeting showcasing international respiratory research from all over the globe. The Canadian Thoracic Society (CTS) was prominent in the organization and presentation, and deserves to feel proud of the meeting's success; the Society's annual dinner was well and enthusiastically attended. At the dinner, Dr Clarence Guenter gave the Christie Lecture, which will appear in the Journal before the end of the year.

For me, the most heartening impression was of the excitement and enthusiasm of younger colleagues in their research and clinical endeavours; there was little postrestructuring depression. They were all clearly looking forward to the future with considerable confidence, at least in part because of the promise of improved funding opportunities, and incentives for collaboration ('networking') afforded by the Canadian Institutes of Health Research (CIHR), described in our first issue of the year by Dr Malcolm King (Can Respir J 2000;(7)1:17-18).

At the meeting, there was much interest shown in the Journal, and it proved impossible to keep up with the demand for (free) copies at the Lung Association stand. As is our habit, we took the opportunity at the CTS annual meeting to meet with the editorial board of the Journal. These meetings are always held over a lunch hosted by Pulsus Group, and are invariably a pleasant occasion. I find them particularly valuable, because they allow members of the board to provide their feedback about the Journal, and to generate and discuss ideas for new features and problem solving. Some new features introduced in the past year (Classics Revisited, Clinical Vignettes and the Page for Patients) could do

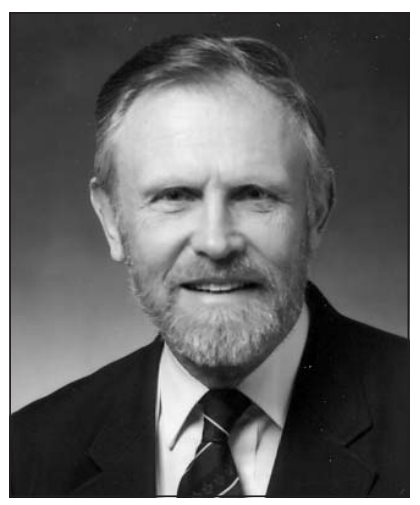
with some feedback from readers of the Journal. The attraction of regular reviews, particularly of advances in understanding and treatment and bridging basic science to clinical respirology, was emphasized by several board members. Dr Jim Hogg suggested these be authored by senior fellows in academic centres, be reviewed by their mentor and the author awarded a scholarship of $\$ 500$. Because this resolution was adopted, I hope that prospective contributors and/or heads of departments will call me with suggestions.

Also at the editorial board meeting, Mr Robert Kalina, publisher, announced that Pulsus Group would be making its largest annual donation to the CTS. This is a proportion of the advertising and subscription revenues generated by the Journal and can be considered an indirect measure of the health of the Journal.

Finally at the meeting, I indicated my intention to resign as Editor-in-Chief of the Journal at some time in the coming 18 months. A search strategy is in place, and the position will shortly be advertised. Anyone with an interest in this rewarding opening is encouraged to call me and discuss any queries they may have.

Norman $L$ Jones Editor-In-Chief Canadian Respiratory Journal 


\section{Du plaisir pour tous!}

A u moment d'écrire ces lignes, le congrès de l'American Thoracic Society, édition 2000, qui s'est tenu à Toronto, est terminé déjà depuis une semaine. Le congrès semble avoir remporté un franc succès à tous les points de vue. Malgré les inconvénients associés aux congrès de grande envergure, dont quelques séparations ennuyeuses entre les diverses séances, nul ne peut se plaindre du caractère exhaustif de la couverture du sujet avec plus de 6000 diaporamas et présentations par affiches. J'ai particulièrement été impressionné par le nombre de chercheurs chevronnés qui y ont participé et qui ont montré avec force qu'ils n'étaient pas de simples noms du passé, mais qu'ils avaient beaucoup à offrir aux chercheurs universitaires, particulièrement sur le plan d'égal à égal. On pouvait donc se trouver en face d'une présentation par affiches de l'un des ces grands noms : Sol Permutt, Bob Hyatt, John West, Ann Woolcock, Neil Pride et de nombreux autres qui ont offert généreusement leur temps, leur expérience et leur sagesse. Il ne peut y avoir de meilleur congrès pour exposer la recherche qui s'effectue sur les maladies respiratoires à l'échelle internationale. La Société canadienne de thoracologie (SCT) a été un acteur très important de l'organisation et de la présentation du congrès, et mérite d'être fière du succès que celui-ci a remporté; le dîner annuel de la Société a également été fort prisé. Le $\mathrm{D}^{\mathrm{r}}$ Clarence Guenter y a présenté la conférence Christie, qui sera publiée dans le Journal d'ici la fin de l'année.

Personnellement, ce qui m'a le plus frappé a été l'enthousiasme dont ont fait preuve de jeunes collègues dans leurs recherches et leurs travaux cliniques, les effets de la restructuration ne les ayant pas trop touchés. De toute évidence, ils envisagent l'avenir avec un optimisme débordant, en raison, du moins, des possibilités accrues de financement et des mesures incitatives à la collaboration (« réseautage ») qu'offrent les Instituts canadiens de recherche en santé (ICRS) et qui ont été décrites par le $\mathrm{D}^{\mathrm{r}}$ Malcolm King dans le premier numéro de l'année ( $J$ canadien de pneumologie 2000;(7)1;17-18).

Durant l'événement, les congressistes ont montré un vif intérêt pour le Journal, au point où il a été impossible de répondre à la demande d'exemplaires offerts gratuitement au stand de l'Association pulmonaire. Comme d'habitude, nous avons profité de l'occasion pour rencontrer les membres du comité de rédaction du Journal. Les déjeuners de travail sont toujours offerts par le Pulsus Group et sont immanquablement agréables. J'estime que ces rencontres sont particulièrement précieuses parce qu'elles permettent aux membres du comité de formuler leurs commentaires sur le Journal, de présenter des idées de nouveaux articles et de résoudre des problèmes. Il serait intéressant

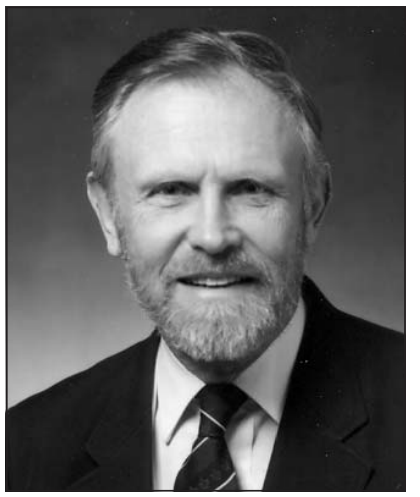
de recevoir des commentaires des lecteurs sur les nouveautés présentées l'année dernière («Classic Revisited », «Clinical Vignettes » et « Page for Patients »). Plusieurs membres du comité ont souligné l'attrait des chroniques, notamment celles qui portent sur les progrès réalisés dans la compréhension et le traitement des maladies respiratoires et qui permettent d'établir un lien entre la science pure et la pneumologie clinique. Le $\mathrm{D}^{\mathrm{r}} \mathrm{Jim}$ Hogg a suggéré que les articles soient rédigés par des agrégés supérieurs de recherche rattachés à des centres universitaires, qu'ils soient révisés par leur mentor et que l'auteur reçoive une bourse de 500 \$. Comme la résolution a été adoptée, j’espère que les collaborateurs éventuels ou des directeurs de département me feront part de leurs suggestions.

De plus, à la réunion du comité de rédaction, monsieur Robert Kalina, éditeur, a annoncé que le Pulsus Group ferait son don annuel le plus important à la SCT. Il s'agit d'une partie des revenus de publicité et d'abonnement du Journal, et ce don peut être considéré comme un reflet de la santé du Journal.

Enfin, au cours de la réunion, j'ai fait part de mon intention de démissionner à titre de rédacteur en chef du Journal au cours des 18 prochains mois. Une stratégie de recherche est en place et le poste sera bientôt affiché. Toute personne intéressée à occuper ce poste très enrichissant est invitée à communiquer avec moi pour obtenir de plus amples s renseignements.

Norman L Jones

Rédacteur en chef Journal canadien de pneumologie 


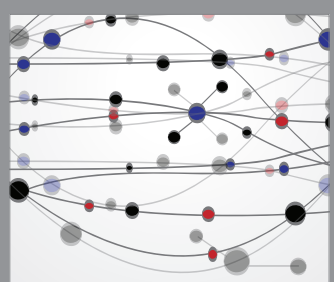

The Scientific World Journal
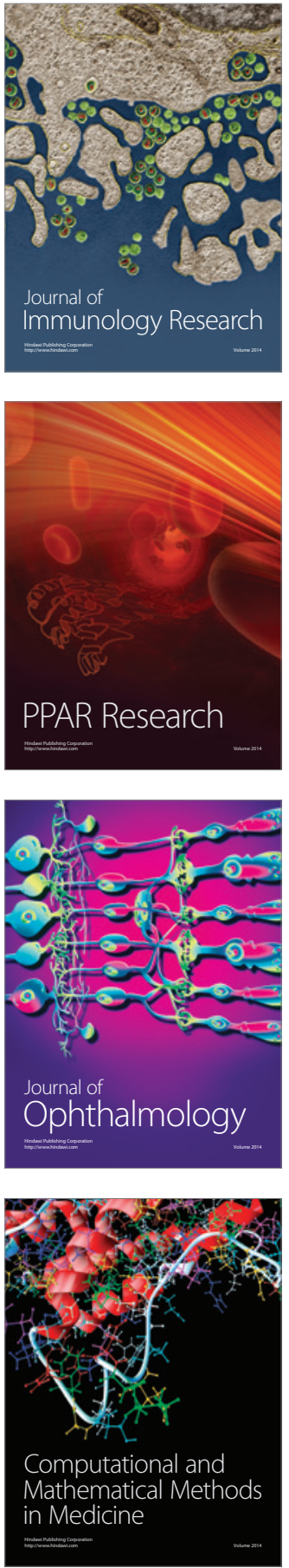

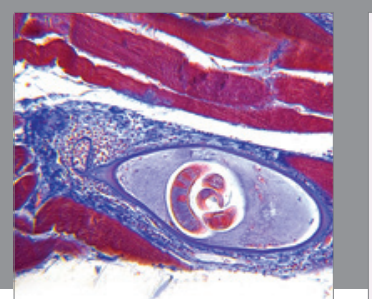

Gastroenterology Research and Practice

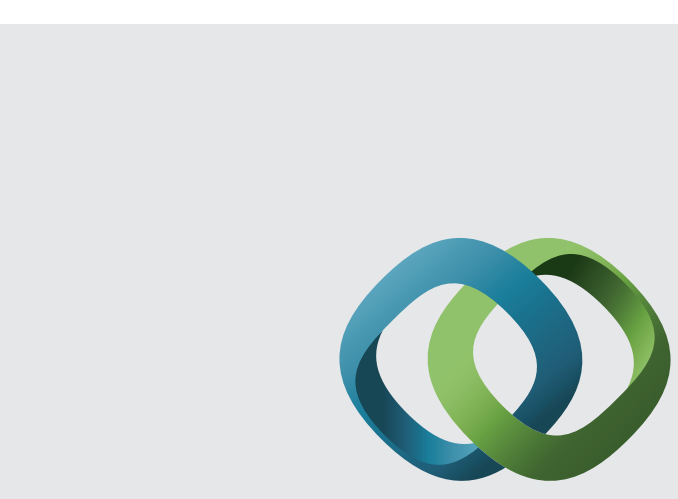

\section{Hindawi}

Submit your manuscripts at

http://www.hindawi.com
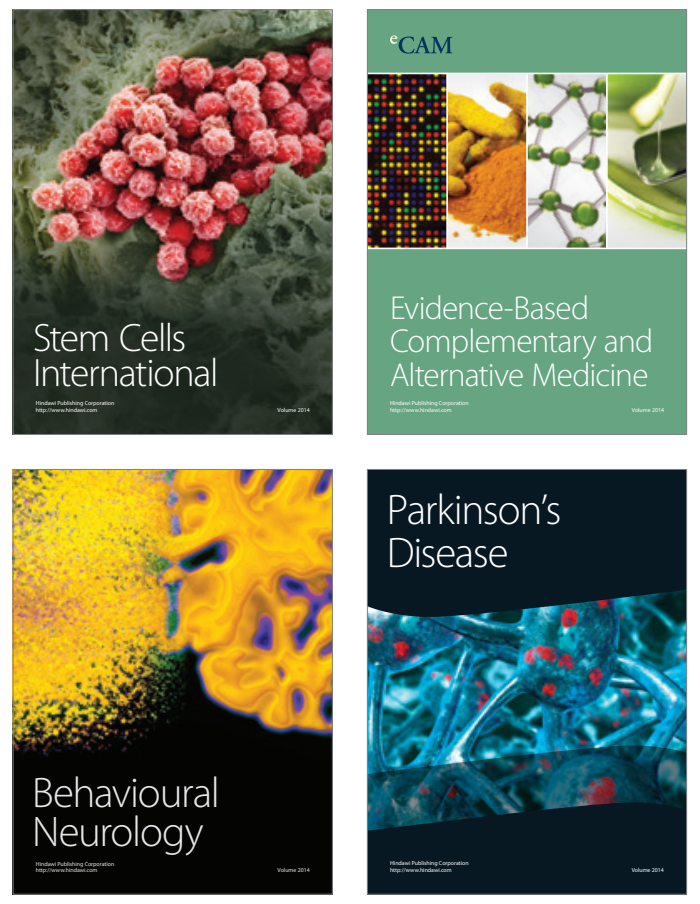
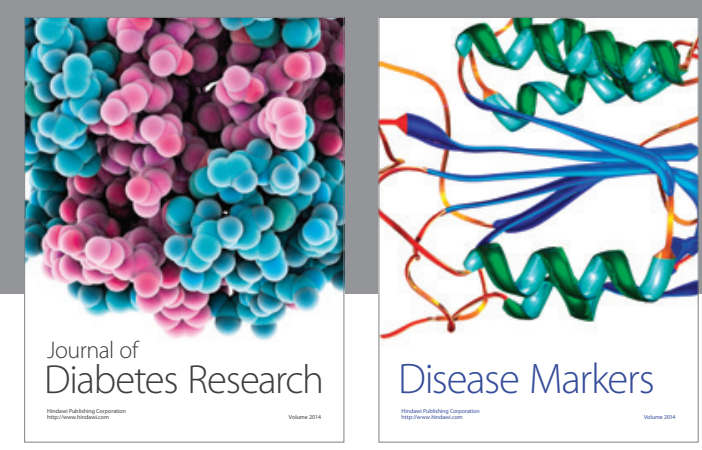

Disease Markers
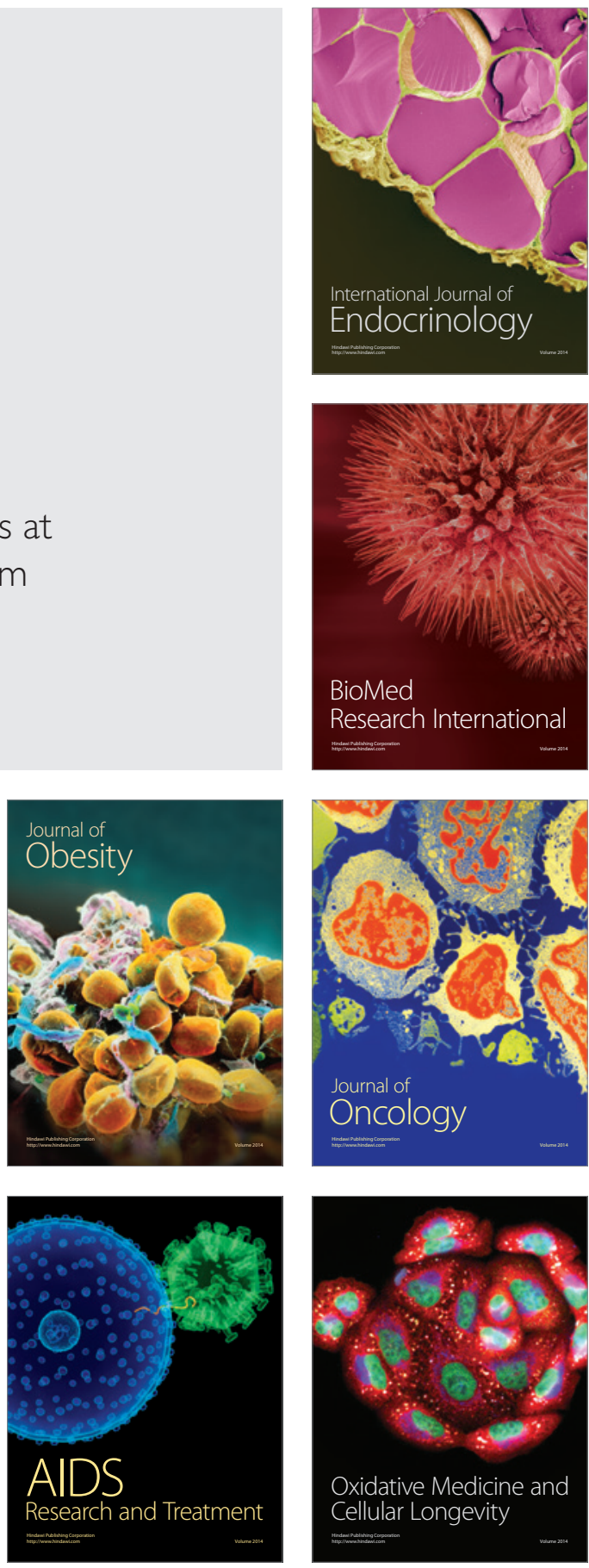